\title{
The robustness of the permeability of constructal tree-shaped fissures
}

\author{
M. Alalaimi ${ }^{a}$, S. Lorente ${ }^{\mathrm{b}}, \mathrm{W}$. Wechsatol ${ }^{\mathrm{c}}$, A. Bejan $^{\mathrm{a}, *}$ \\ ${ }^{a}$ Department of Mechanical Engineering and Materials Science, Duke University, Box 90300, Durham, NC 27708-0300, USA \\ ${ }^{\mathrm{b}}$ Université de Toulouse, INSA, 135 Avenue de Rangueil, Toulouse 31077, France \\ 'King Mongkut's University of Technology Thonburi, 126 Pracha-Utid Rd, Bangmod, Thungkru, Bangkok 10140, Thailand
}

\section{A R T I C L E I N F O}

\section{Article history:}

Received 3 April 2015

Received in revised form 10 June 2015

Accepted 14 June 2015

Available online 4 July 2015

\section{Keywords:}

Constructal

Fissures

Permeability

Porosity

Fracture

Tree-shaped flow

\begin{abstract}
A B S T R A C T
Here we develop analytically the formulas for effective permeability in several configurations using the closed-form description of tree networks designed to provide flow access. The objective was to find the relation between the permeability and porosity of tree-shaped fissures. We found the effect of the fracture size on the permeability for fixed number of bifurcation and the results showed that the permeability of the fracture network increased rapidly with the size of the fracture. Next, we found a relation between the Reservoir Quality Index (RQI) and the porosity of the fracture. The results in this paper have been validated by comparison with experimental and numerical results. We show that the permeability formulas do not vary much from one tree design to the next, suggesting that similar formulas may apply to naturally fissured porous media with unknown precise details, which occur in natural reservoirs.
\end{abstract}

(c) 2015 Elsevier Ltd. All rights reserved.

\section{Multi-scale flow structures distributed non uniformly}

Fractured porous media such as hot dry-rock deposits [1-3] are considerably more complicated and difficult to describe than the packed-spheres systems described by compact formulas such as the Carman-Kozeny permeability model [4]. The reason is that fractured media have fissures with many length scales, which are distributed and oriented nonuniformly through the available volume. The fluid that is forced to permeate through such a medium follows paths that can be described as random combinations of organized channel flow and disorganized porous medium flow. The organized portion is the flow through the largest fissures, while the disorganized flow is the seepage through the smaller volumes filled with the finer fissures.

The challenge is to describe the permeability of such complex flow structures by means that approach the simplicity of the models known for uniform packings of particles with a single length scale. Here we show that the flow permeability of fractured solid has relatively universal and predictable characteristics. We show this by modeling the multi-scale flow volume as tree shaped, with a precise architecture that results from constructal design: the maximization of flow access through the fluid space $[5,6]$.

A new trend in convection and designed porous media is the use of tree-shaped structures for accessing areas or volumes with minimal flow resistance. The current progress was reviewed in Ref. [7].

\footnotetext{
* Corresponding author.

E-mail address: abejan@duke.edu (A. Bejan).
}

One development that is relevant to the present paper is the design of nearly-optimal dendritic structures based on compact recurrence formulas obtained by minimizing the length of every link in the dendritic flow structure [8]. Another relevant development is the demonstrated robustness [9] of dendritic flow configurations: the performance level does not vary significantly from one configuration to another.

The current progress made on dendritic structures is an opportunity to describe in compact form the 'volumetric permeability' of such structures. This may shed light on the constitution of the effective permeability of a naturally fractured porous medium. The basis for this speculation is that dendritic flow structures are similar to fissured porous media. They have channels with multiple length scales, which are organized hierarchically. These channels are distributed and oriented nonuniformly. The robustness of dendritic structures suggests that the performance of similarly complex structures (e.g., fissured media) is similar to that of dendritic structures, and may be expressed by formulas developed for dendritic structures. Progress in this direction was demonstrated by $\mathrm{Xu}$ et al. [10] who calculated the permeability of a fractal-like tree network in one configuration, fractal-like tree networks between one point and a straight line. A fractal-like tree is a geometric object with postulated (assumed, not predicted) algorithm of construction and smallest length scale. Several other investigators have studied self-similar fracture networks. For example, Velde et al. and Sahimi et al. [11-14] analyzed the fractal patterns of fractures in porous media. Gueguen and Chelidze [15] also studied the fracture patterns in Stockbridge dolomite marble, 


\section{Nomenclature}

D channel thickness, $\mathrm{m}$

$i \quad$ bifurcation level

$K \quad$ permeability, $\mathrm{m}^{2}$

$L \quad$ channel length, $\mathrm{m}$

$L_{d} \quad$ distance from the center to the side of the configuration, $\mathrm{m}$

$\dot{m} \quad$ mass flow rate, $\mathrm{kg} / \mathrm{s}$

$P \quad$ pressure, $\mathrm{Pa}$

RQI Reservoir Quality Index

$u \quad$ volume average velocity, $\mathrm{m} / \mathrm{s}$

$V \quad$ volume, $\mathrm{m}^{3}$

W channel width, $\mathrm{m}$

Greek symbols

$\theta \quad$ angle, degree

$\phi \quad$ porosity

which reported a highly interconnected and very branched network. Wang et al. [16] and Voorn et al. [17] reported numerically the permeability of fractured porous media. Miao et al. [18] studied analytically the permeability of fractured rocks.

In this paper we explore the theoretical basis for the permeability of fissures by taking full advantage of the closed-form description of tree networks with minimal-length channels [8]. We develop analytically the formulas for effective permeability in four different flow configurations: tree connecting one point and a square frame, flow perpendicular to the plane of the tree, tree connecting a circle with its center, and tree connecting a point with an area. To study the effect of changing the flow direction (along the plane of the design or normal to the plane) is important because the assembly of fissures is not necessarily the result of the flow itself. Fissures in rocks are not like the river channels on the plain. They can be of solid mechanics origin, such as the phenomenon of cracking during the release of tension in a solid that is in a state of volumetric tension. We show that the permeability formulas do not vary much from case to case, suggesting that essentially the same broad formula applies to naturally fissured porous media.

\section{Tree connecting one point and a square frame}

Start with is the two-dimensional tree structure shown in Fig. 1. The flow connects the center of the square with many ports positioned equidistantly on the perimeter. Each channel is modeled as the space between two parallel plates of spacing $D_{i}$ and flow length $L_{i}$, where $i$ indicates the pairing or bifurcation level. The smallest scale is found near the perimeter, $i=0$. The structure has $\mathrm{n}$ levels of pairing. The largest channels reach the center, $i=n$. Symmetry allows us to focus on a one-eighth section of the structure.

The optimal configuration is discovered numerically and sketched qualitatively as Section 2in Fig. 1: noteworthy is the longest channel, which is not aligned with the diagonal of the square. The alternative shown as Section 1 is the structure based on minimizing the length of each channel, starting from the smallest $\left(L_{0}\right)$ and proceeding towards the largest [8]. This structure is characterized by the relations

$L_{i}=2^{i} L_{0}$

$n_{i}=2^{n-i}$

$\dot{m}_{i}^{\prime}=2^{i} \dot{m}_{0}^{\prime}$

$\begin{array}{ll}\rho & \text { density, } \mathrm{kg} / \mathrm{m}^{3} \\ v & \text { kinematic viscosity, } \mathrm{m}^{2} / \mathrm{s} \\ \mu & \text { dynamic viscosity, } \mathrm{N} \mathrm{s} / \mathrm{m}^{2}\end{array}$

Subscripts

c center

$n \quad$ largest scale

$p \quad$ pore

$t$ total

0 smallest scale

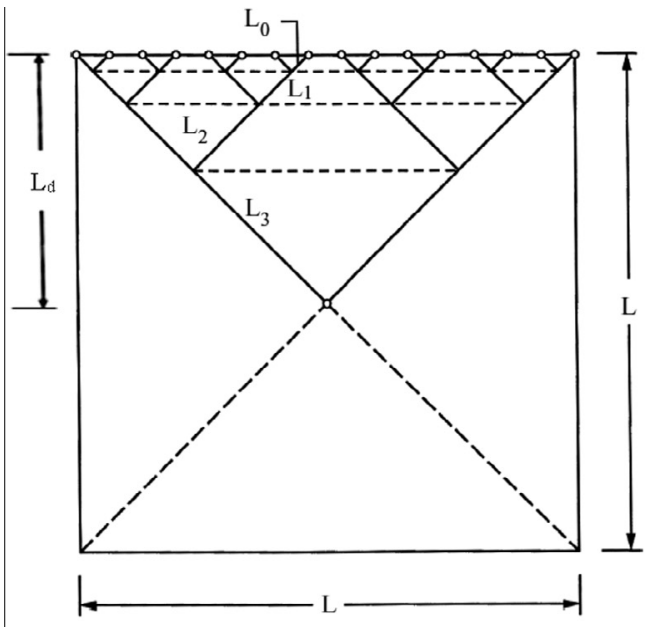

(I)

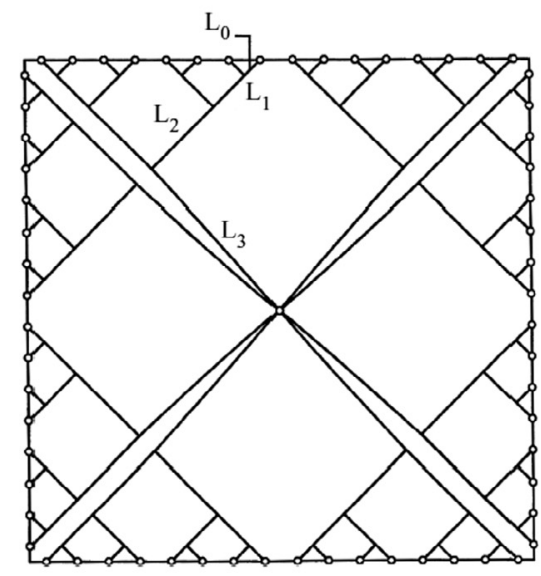

(II)

Fig. 1. Tree-shaped network connecting a square frame with its center.

where $i=0,1, \ldots, n$. Furthermore, $n_{i}$ is the number of channels of size $\left(D_{i}, L_{i}\right)$, and $\dot{m}_{i}^{\prime}$ is the mass flow rate $\left(\mathrm{kg} \mathrm{s}^{-1} \mathrm{~m}^{-1}\right)$ through a channel at level $i$. The number of ports on one eighth of the square 
perimeter is $n_{0}=2^{n}$. The distance from the center to the side of the square is $L_{d}=2^{-1 / 2}\left(2^{n+1}-1\right) L_{0}$.

The necessary recurrence relation for $D_{i}$ follows from the minimization of flow resistance subject to fixed total channel volume. We assume that each channel is small and slender enough that the flow is in the Poiseuille regime. It can be shown [7] that a Y-shaped structure formed by two $D_{i}$ channels merging into one $D_{i+1}$ channel has minimal overall flow resistance when $D_{i+1} / D_{i}=2^{1 / 2}$, hence

$D_{i}=2^{i / 2} D_{0}$

The flow resistance between the center of the square and the total flow rate is $\Delta P / \dot{m}_{n}$, where $\Delta P$ is the overall pressure difference,

$\Delta P=\sum_{i=0}^{n} \Delta P_{i}$

The pressure drop along a channel of level $i$ is $\Delta P_{i}=12 v \dot{m}_{i}^{\prime} L_{i} / D_{i}^{3}$, and Eq. (5) becomes

$\Delta P=12 v \dot{m}_{0}^{\prime} \frac{L_{0}}{D_{0}^{3}} \frac{2^{(n+1) / 2}-1}{2^{1 / 2}-1}$

From this we can deduce the effective permeability of the structure by writing the Darcy law

$u=\frac{K}{\mu} \cdot \frac{\Delta P}{L_{d}}$

where $L_{d}$ is the distance from the center to the side of the square, and $u$ is the volume averaged velocity of the flow crossing the $L_{d}$ boundary of Section 1 in Fig. 1 (note that we analyze the flow on $1 / 8$ th of the square domain):

$u=\frac{\dot{m}_{n}^{\prime}}{\rho L_{d}}$

Eqs. (6)-(8) yield the permeability as a function of the smallest scales $\left(D_{0}, L_{0}\right)$ and complexity $(n)$ :

$K=\frac{2^{n}\left(2^{1 / 2}-1\right) D_{0}^{3}}{12\left[2^{(n+1) / 2}-1\right] L_{0}}$

The porosity $(\phi)$ of porous medium is the ratio between the pore volume $V_{p}$ to the total volume $V_{t}$. The total porosity can be defined as $\phi=V_{p} / V_{t}$.

The Carman-Kozeny relation for the permeability of packed spheres shows $K$ as a function of the particle diameter and the porosity of the structure. The porosity of the half system described by Eq. (9) is

$\phi=\frac{2^{n+2}\left[2^{(n+1) / 2}-1\right] D_{0}}{\left(2^{1 / 2}-1\right)\left(2^{n+1}-1\right)^{2} L_{0}}$

By eliminating $L_{0}$ between Eqs. (9) and (10) we find

$K=\phi D_{0}^{2} \frac{\left(2^{1 / 2}-1\right)^{2}\left(2^{n+1}-1\right)^{2}}{48\left(2^{(n+1) / 2}-1\right)^{2}}$

Alternatively, this result can be expressed in terms of the thickness of the largest channel, $D_{n}$,

$K=\phi D_{n}^{2} \frac{\left(2^{1 / 2}-1\right)^{2}\left(2^{n+1}-1\right)^{2}}{24 \cdot 2^{n}\left[2^{(n+1) / 2}-1\right]^{2}}$

Eqs. (11) and (12) resemble the Carman-Kozeny formula $K=\phi^{3} D^{2} /\left[180(1-\phi)^{2}\right]$, where $D$ is the particle diameter, but in addition it shows the effect of complexity $(n)$. These $K$ formulas

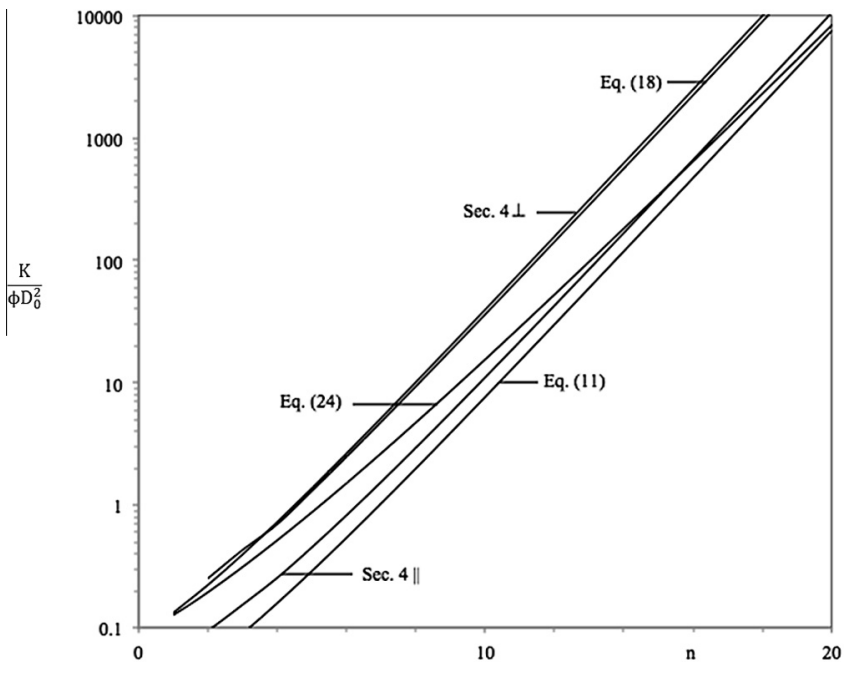

Fig. 2. The effective permeability group referenced to the smallest scale, as a function of complexity $(n)$.

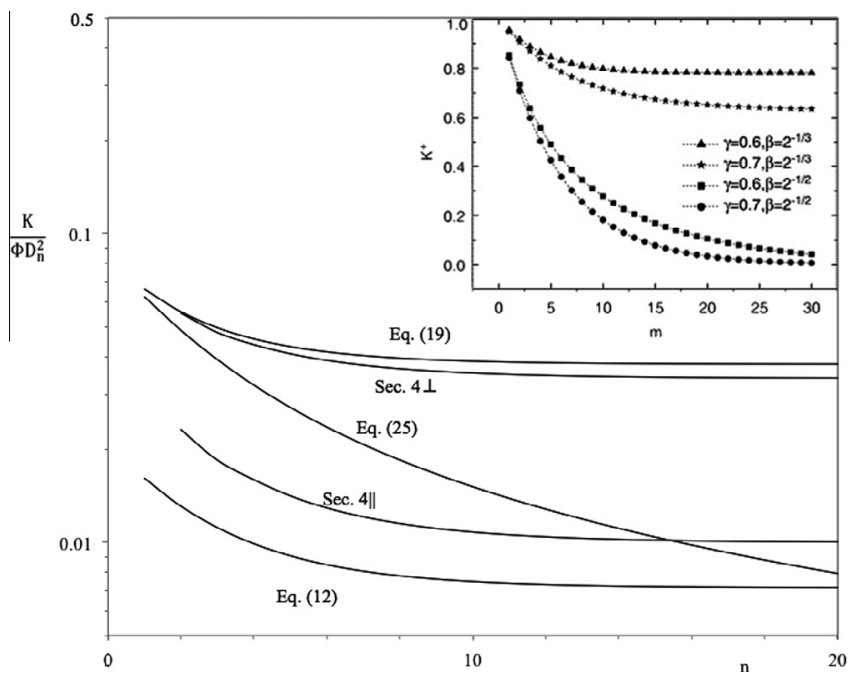

Fig. 3. The effective permeability group referenced to the largest scale, as a function of complexity $(n)$, where the inset shows results from Ref. [10].

are plotted as $K / \phi D_{0}^{2}$ and $K / \phi D_{n}^{2}$ in Figs. 2 and 3. An important first conclusion is that Eq. (12) is the more appropriate form of presenting the result, because $K / \phi D_{n}^{2}$ becomes a constant when $n$ is large enough.

\section{Flow perpendicular to the plane of the tree}

In the analysis of Fig. 1 it was assumed that the flow is aligned with the $L_{i}$ lengths, which is the direction of the flow for which the tree structure with minimal global resistance was generated. In a natural multi-scale flow structure, however, the flow direction is governed by the applied pressure difference, not by the fracture mechanism that generated the structure. Consequently, an additional idea to explore is whether the flow direction has a major effect on the effective permeability of the complex structure.

Assume that in Fig. 1 the flow is oriented in the direction perpendicular to the figure. In that direction, the dimension of the one-eighth section is $W$, which is sufficiently larger than $L_{n}$. Each channel is a space of thickness $D_{i}$, width $L_{i}$, flow length $W$, and 
pressure drop $\Delta P$, and mass flow rate $\dot{m}_{i}\left(\mathrm{~kg} \mathrm{~s}^{-1}\right)$ The total mass flow rate through this system is

$\dot{m}=\sum_{i=0}^{n} n_{i} \dot{m}_{i}$

where for Poiseuille flow

$\dot{m}_{i}=\frac{\Delta P D_{i}^{3} L_{i}}{12 v W}$

By combining Eqs. (13) and (14) we obtain the global relation between pressure difference and mass flow rate,

$\dot{m}_{i}=\frac{\Delta P D_{0}^{3} L_{0}}{12 v W} 2^{n} \frac{2^{3(n+1) / 2}-1}{2^{3 / 2}-1}$

The corresponding permeability of this structure comes from the Darcy relation

$u=\frac{K}{\mu} \frac{\Delta P}{W}$

in which the volume averaged velocity is

$u=\frac{\dot{m}}{\rho L_{d}^{2} / 2}$

Together, Eqs. (15)-(17) and the porosity formula (10) yield

$K=\phi D_{0}^{2} \frac{2^{1 / 2}-1}{12\left(2^{3 / 2}-1\right)} \frac{2^{3(n+1) / 2}-1}{2^{(n+1) / 2}-1}$

$K=\phi D_{n}^{2} \frac{2^{-n}\left(2^{1 / 2}-1\right)}{12\left(2^{3 / 2}-1\right)} \frac{2^{3(n+1) / 2}-1}{2^{(n+1) / 2}-1}$

Eqs. (18) and (19) are plotted in Figs. 2 and 3. They show once again that the use of $D_{n}^{2}$ is a more appropriate way of accounting for the effect of fissure size, because $K$ becomes independent of $n$ when $n$ is larger. Another conclusion is that the change in flow direction from along the tree of Fig. 1 to perpendicular to the plane of Fig. 1 has practically no effect on the behavior of the permeability function $K\left(\phi, D_{n}^{2}, n\right)$.

\section{Tree connecting a circle with its center}

Additional evidence on the robustness of the $K$ function for multi-scale fissured media is provided by the analysis of Poiseuille flow through point-circle networks of the type shown in Fig. 4. The nomenclature is the same as in Fig. 1, with one additional degree of freedom: the number of the largest channels $\left(D_{n}\right)$ that reach the center $\left(n_{c}\right)$. In Fig. 4 that number is $n_{c}=3$. For the point-circle trees of Fig. 4, Ref. [19] reported the complete architectures for minimal flow resistance for cases with $3 \leqslant n_{c} \leqslant 16$ and bifurcation levels in the range $0 \leqslant n \leqslant 4$.

The optimal values for the dimensions of each channel $\left(D_{i}, L_{i}\right)$ are reported in tabular form Ref. [19], with the reminder that $i=n$ represents the smallest dimensions, at the periphery, and $i=0$ the dimensions of the largest channels that reach the center (note that the present $i$ notation is the reverse-direction relative to the notion used in Ref. [19]). Nearly optimal versions of the same structures are reported in Ref. [8] based on the same channel length minimization algorithm that generated Section 1 of Fig. 1. In this paper we relied on the minimal-length structures, but for conciseness we fixed the number of central channels at $n_{c}=3$, as in the example shown in Fig. 4.

For the case where the flow is oriented along the tree (i.e., in the plane of Fig. 4) the analysis of Section 2 continues to apply, provided that $L_{d}$ is replaced by the circle radius $R$. The radius $R$

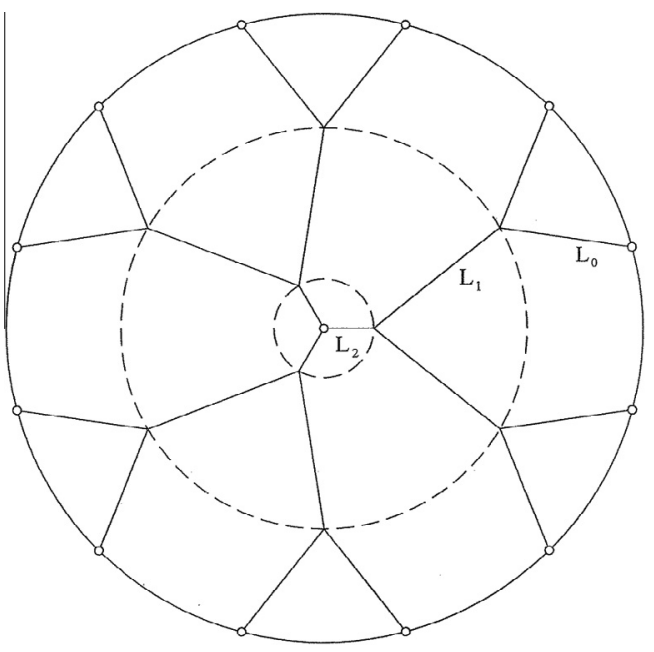

Fig. 4. Tree-shaped network connecting a circle with its center.

can be represented in terms of $L_{0}$ by using the values of $L_{i}$ reported in Ref. [19]. Let the ratios between the channels length $L_{1} / L_{0}=a$, $L_{2} / L_{1}=b, L_{3} / L_{2}=c \ldots$, where $a, b$ and $c$ are values that can be found from the table in Ref. [19]. The angle $\theta_{i}$ for each level of bifurcation is also reported in Ref. [19]. Therefore, the radius of the circle is

$R=\left[1+a \cos \theta_{1}+a b \cos \theta_{2}+a b c \cos \theta_{3}+\cdots\right] L_{0}$

$\phi=\frac{V_{p}}{V_{t}}=\frac{\sum_{i=0}^{n} n_{i} L_{i} D_{i}}{\pi R^{2}}$

By evaluating the permeability for each level of pairing $(n)$ using the values of the angles and ratios tabulated in Ref. [19] we find the permeability as a function of the smallest scales $\left(K / \phi D_{0}^{2}\right)$ and the thickness of the largest channel $\left(K / \phi D_{n}^{2}\right)$. These results are presented in Figs. 2 and 3, where they are labeled Section 4\|. The results reveal the robustness of $K$, because the change from square systems (Fig. 1) to round systems (Fig. 4) has no effect on the behavior of the function $K\left(\phi, D_{n}^{2}, n\right)$.

If we assume that the flow is perpendicular to the plane of Fig. 4, the analysis of Section 3 can be repeated to obtain the permeability as a function of the smallest scales $\left(K / \phi D_{0}^{2}\right)$ and the permeability in terms of the thickness of the largest channel $\left(K / \phi D_{n}^{2}\right)$. These results are reported in Figs. 2 and 3, where they are labeled Section $4 \perp$. These results reinforce the earlier conclusions, including the insensitivity of $K$ to the change in flow direction. Furthermore, more $K$ curves could be derived for other tree-shaped arrangements of fissures, and it is very likely that they would look similar to what we have seen here in Figs. 2 and 3. One class of tree architectures that would produce such a set of $\mathrm{K}$ curves are the point-circle trees optimized in Ref. [19]. Another class if analyzed in the next section.

\section{Tree connecting a point with an area}

The distinguishing feature of the tree structures analyzed until now is that each connects on point with many points positioned equidistantly on a line (square Fig. 1, and circle Fig. 4). Another class that has received considerable attention are the two-dimensional trees that connect a point with a finite-size area. In this case, the many points of the tree canopy are spread uniformly over an area. Fig. 5 shows a 4-point canopy, which is connected with the tree root by a dichotomous flow structure. The flow in this case is laminar. The smallest scales are $\left(D_{0}, L_{0}\right)$. 


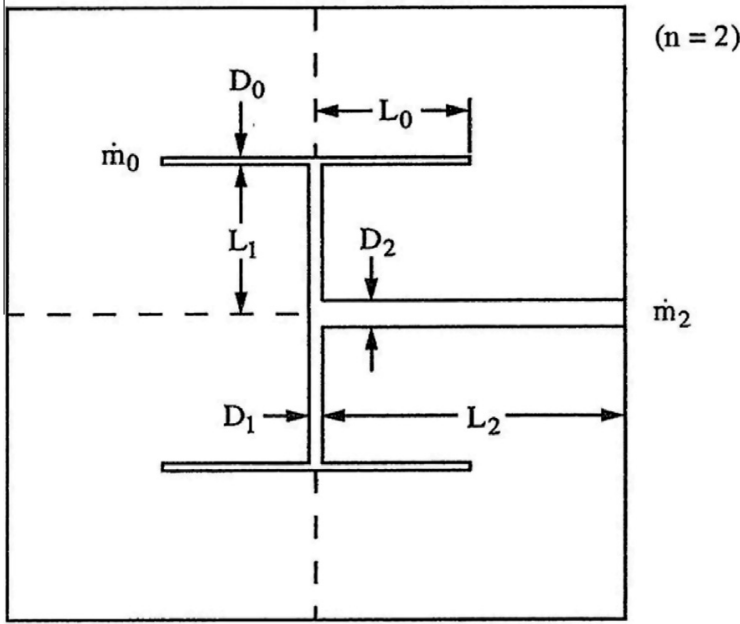

Fig. 5. Tree-shaped network connecting an area with a point.

In Fig. 5 there are only two levels of pairing or bifurcation, $n=2$. The nomenclature is the same as in Section 3, with the observation that the flow is perpendicular to the plane of Fig. 5. In that direction the system dimension is $W$.

The construction of Fig. 5 is detailed in Ref. [7], where the constructal principle is the minimization of flow resistance at each level of assembly, or pairing. This design approach has shown that the shape of each new larger construct alternates between a square and a rectangle formed by two squares. For this reason, the largest length of each new construct doubles after every two levels of pairing. For example, starting from the elemental square $L_{0} \times L_{0}$ in Fig. 5, the length of the largest channel that reaches the root progresses in the sequence $L_{0}, L_{1}=L_{0}, L_{2}=2 L_{0}, \ldots$. When the tree structure has a large number of pairing levels $n$, it is convenient to approximate the sequence of length scales as

$L_{i} \cong 2^{i / 2} L_{0} \quad(i=0,1, \ldots, n)$

It can be shown that the structure formed by two $D_{i}$ channels merging into one $D_{i+1}$ channel has minimal overall flow resistance when $D_{i+1} / D_{i}=2^{1 / 2}$, hence $D_{i}=2^{i / 2} D_{0}$. The porosity of the two-dimensional flow structure is equal to the area of the tree channels divided by the total frontal area of the system $2^{n+2} L_{0}^{2}$. The pore volume is $2^{n} D_{0} L_{0}(n+1)$. The porosity estimated in this manner is

$\phi=\frac{1}{4}(n+1) \frac{D_{0}}{L_{0}}$

The effective permeability follows from Eqs. (15)-(17) and (23), with the observation that in place of $L_{d}^{2} / 2$ in Eq. (17) we use the frontal area $2^{n+2} L_{0}^{2}$. The result can be reported in two ways

$K=\phi D_{0}^{2} \frac{2^{n+1}-1}{12(n+1)}$

$K=\phi D_{n}^{2} \frac{2^{-n}\left(2^{n+1}-1\right)}{12(n+1)}$

and is plotted in Figs. 2 and 3. We see once again the use of $D_{n}^{2}$ instead of $D_{0}^{2}$ is more effective. The new feature is that the effect of the increasing complexity $(n)$ is not negligible, cf. Fig. 3.

\section{Discussion}

In the analysis presented above we showed the robustness of the permeability $K$ function for multi-scale fissures. At this stage it is important to validate the dendritic flow structure method used in this paper with numerical and experimental results based on conventional methods. Fig. 3 showed the effect of the number of bifurcation $\mathrm{n}$ on the permeability, and indicated that the effective permeability becomes constant when $n$ is large enough. This is in agreement with results of Ref. [10], with the observation that in Ref. [10] $\mathrm{m}$ is the number of bifurcation and $K^{+}$is the dimensionless permeability referenced to the largest scale.

Fig. 6 shows the permeability versus the porosity for different configurations, which are labeled Sections 2-5. The permeability increases with the porosity, and this is in agreement with experimental results from Ref. [20]. Our results also agree with the numerical simulation and experimental results reported by several other authors [18,21-24].

Another result of the present paper is the effect of the fracture size $D_{n}$ on the permeability for different configurations. Fig. 7 shows the effect of size on the permeability in the configurations presented in Figs. 1, 4 and 5 for a fixed number of bifurcations, $n=4$. The figure shows that the permeability of the fractured solid depends strongly on the largest scale $D_{n}$. The results show agreement with the experimental data reported in Ref. [25]. By repeating the analysis by changing the number of bifurcations $(n)$ we found that the behavior shown in Fig. 7 of the permeability relation does not change.

The orientation of the flow direction relative to the plane of the fissure configuration has a sizeable effect on the permeability, as shown in Fig 3. For the same configuration, the permeability is higher when the flow is perpendicular to the flow direction. The effect of fracture orientation on the permeability in relatively good agreement with the results from the cubic law method reported in Ref. [18].

An important measure of the permeability of the fracture is the Reservoir Quality Index (RQI), introduced by Amaefule et al. [26]. The RQI is related to the ratio of the permeability to porosity, $\mathrm{RQI}=0.0314(K / \phi)^{1 / 2}$. Fig. 8 shows a log-log plot of porosity versus RQI, which reveals a straight line with unit slope, which agrees with results reported in Refs. [24,26].

In summary, in this paper we determined the permeability of three-shaped arrangements of fissures, which corresponds to dendritic flow structures designed for minimum flow resistance. The width of the largest length scale has a great impact on the

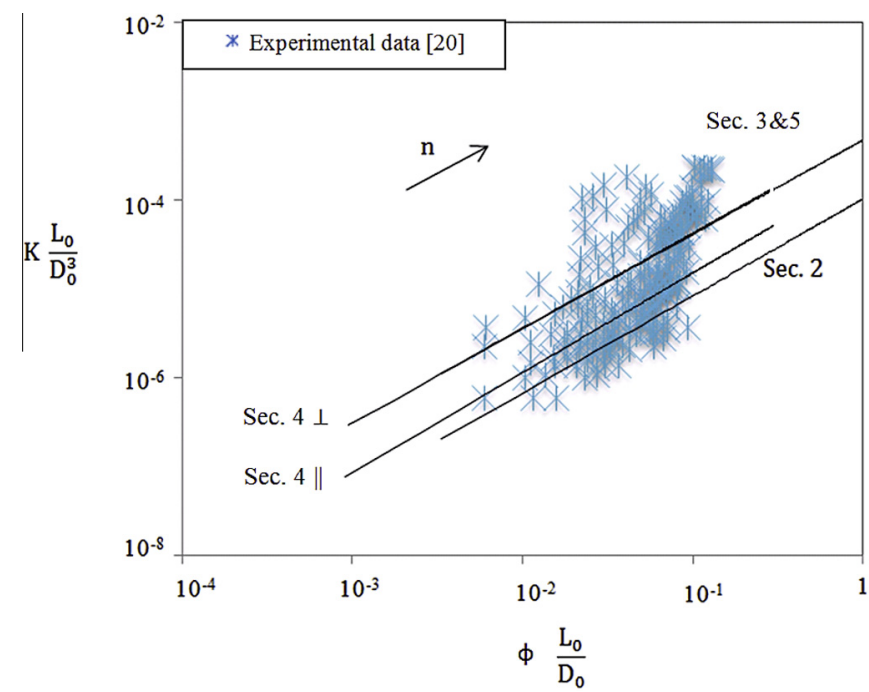

Fig. 6. The permeability versus the porosity of the fissures for different configurations. 


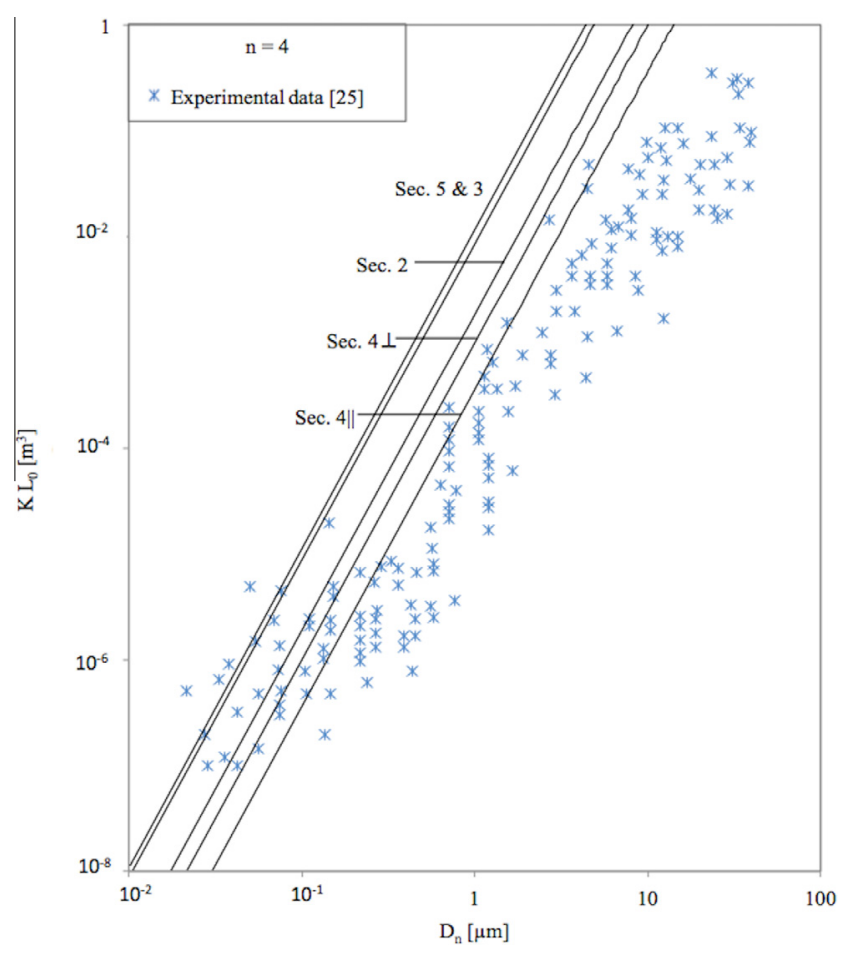

Fig. 7. The effect of the fissure size $D_{n}$ on the permeability.

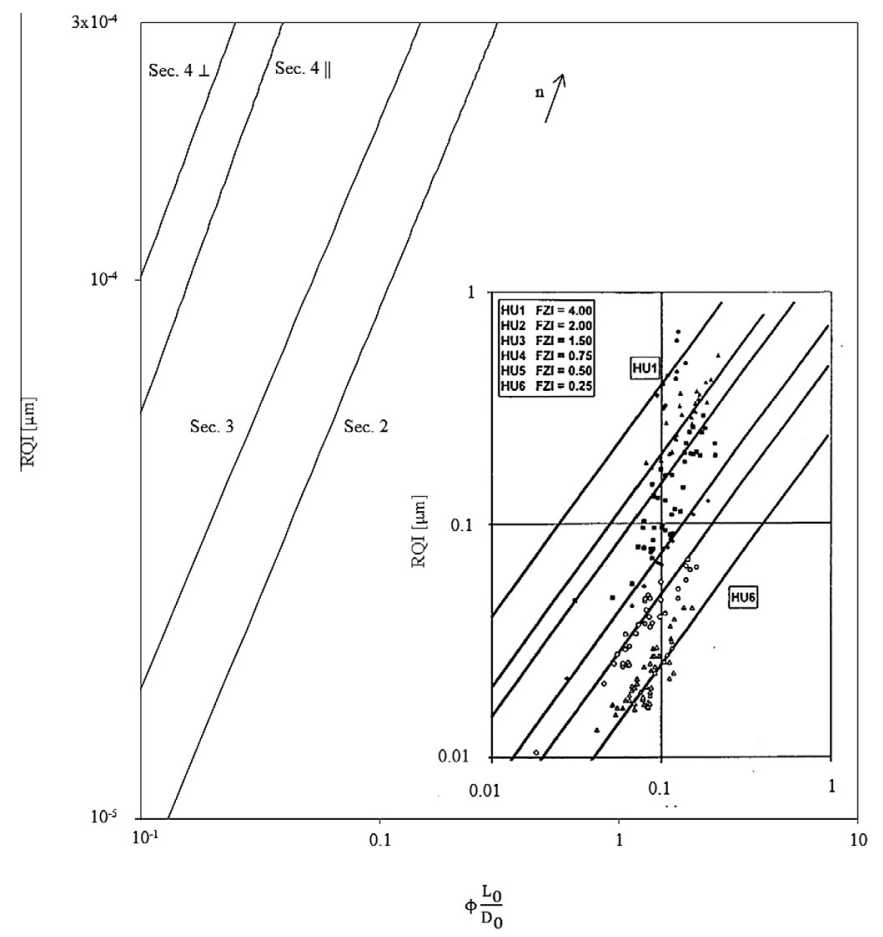

Fig. 8. The relation between the porosity and Reservoir Quality Index (RQI) in a loglog scale, where the inset shows the results from Ref. [26].

permeability than the smallest length scale, as shown in Figs. 1 and 2. As the complexity of the tree-shaped fissure increases, the permeability group becomes constant if referenced to the largest scale $D_{n}$. The results also show that the permeability increases as the porosity increases, as shown in Fig. 6. The permeability of the fracture depends strongly on the largest scale $D_{n}$. The results presented in Fig. 7 shows that as the size of the fracture for a fixed number of bifurcation increases the permeability also increases. Moreover, as the porosity of the fractured medium increases the Reservoir Quality Index increases.

The chief conclusion is that the permeability formulas do not vary much from one configuration to another. The permeability is a robust characteristic of the flow architecture. The practical importance of this conclusion is that the present results apply to other naturally fissured porous media. The approach outlined in this paper can be pursued further by considering other flow regimes, such as developing laminar and transitional to turbulence, especially in view of applications to fissured media at larger scales.

\section{Conflict of interest}

None declared.

\section{Acknowledgment}

Profs. Bejan and Lorente's work was supported by a grant from the US National Science Foundation.

\section{References}

[1] M.K. Hubbert, D.G. Willis, Mechanics of hydraulic fracturing, Trans. AIME 210 (1957) 153-166.

[2] D. Rahm, Regulating hydraulic fracturing in shale gas plays: the case of Texas, Energy Policy 39 (5) (2011) 2974-2981.

[3] M. Marongiu-Porcu, M.J. Economides, S.A. Holditch, Economic and physical optimization of hydraulic fracturing, J. Nat. Gas Sci. Eng. 14 (2013) 91-107.

[4] P.C. Carman, Flow of Gases Through Porous Media, Butterworths, London, 1956.

[5] A. Bejan, Advanced Engineering Thermodynamics, second ed., Wiley, New York, 1997.

[6] A. Bejan, Shape and Structure, from Engineering to Nature, Cambridge University Press, Cambridge, UK, 2000.

[7] A. Bejan, S. Lorente, Design with Constructal Theory, Wiley, Hoboken, 2008.

[8] S. Lorente, W. Wechsatol, A. Bejan, Tree-shaped flow structures designed by minimizing path lengths, Int. J. Heat Mass Transfer 45 (2002) 3299-3312.

[9] W. Wechsatol, S. Lorente, A. Bejan, Tree-shaped networks with loops, Int. J. Heat Mass Transfer 48 (2005) 573-583.

[10] P. Xu, B. Yu, Y. Feng, Y. Liu, Analysis of permeability for the fractal-like tree network by parallel and series models, Physica A 369 (2006) 884-894.

[11] B. Velde, J. Dubois, D. Moore, G. Touchard, Fractal patterns of fractures in granites, Earth Planet. Sci. Lett. 104 (1991) 25.

[12] M. Sahimi, Flow and Transport in Porous Media and Fractured Rock: From Classical Methods to Modern Approaches, second ed., VCH-Wiley, Weinheim, Germany, 2011.

[13] M. Sahimi, M.C. Robertson, C.G. Sammis, Fractal distribution of earthquake hypocenters and its relation to fault patterns and percolation, Phys. Rev. Lett. 70 (1993) 2186.

[14] T.A. Tafti, M. Sahimi, F. Aminzadeh, C.G. Sammis, Use of microseismicity for determining the structure of the fracture network of large-scale porous media, Phys. Rev. E 87 (2013) 032152.

[15] Y. Gueguen, T. Chelidze, Evidence of fractal fracture, Int. J. Rock Mech. Min. Sci. Geomech. Abstr. 27 (1990) 223.

[16] J.-Q. Wang, J.-F. Zhao, M.-J. Yang, Y.-H. Li, W.-G. Liu, Y.-C. Song, Permeability of laboratory-formed porous media containing methane hydrate: observations using X-ray computed tomography and simulations with pore network models, Fuel 145 (2015) 170-179.

[17] M. Voorn, U. Exner, A. Barnhoorn, P. Baud, T. Reuschlé, Porosity, permeability and 3D fracture network characterisation of dolomite reservoir rock samples, J. Pet. Sci. Eng. 127 (2014) 270-285.

[18] T. Miao, B. Yu, Y. Duan, Q. Fang, A fractal analysis of permeability for fractured rocks, Int. J. Heat Mass Transfer 81 (2015) 75-80.

[19] W. Wechsatol, S. Lorente, A. Bejan, Optimal tree-shaped networks for fluid flow in a disc-shaped body, Int. J. Heat Mass Transfer 45 (3-4) (2002) 49114924.

[20] L. Zenga, J. Jiang, Y. Yang, Fractures in the low porosity and ultra-low permeability glutenite reservoirs: a case study of the late Eocene Hetaoyuan formation in the Anpeng Oilfield, Nanxiang Basin, China, Marine Pet. Geol. 27 (7) (2010) 1642-1650.

[21] S. Sadhukhan, D. Mal, T. Dutta, S. Tarafdar, Permeability variation with fracture dissolution: role of diffusion vs. drift, Phys. A: Stat. Mech. Appl. 387 (2008) $4541-4546$.

[22] P. Xu, B. Yu, X. Qiao, S. Qiu, Z. Jiang, Radial permeability of fractured porous media by Monte Carlo simulations, Int. J. Heat Mass Transfer 57 (1) (2013) 369-374. 
[23] R.L. Detwiler, R.J. Glass, W.L. Bourcier, Experimental observations of fracture dissolution: the role of Peclet number on evolving aperture variability, Geophys. Res. Lett. 30 (2003) 1648.

[24] D. Tiab, E.C. Donaldson, Petrophysics: Theory and Practice of Measuring Reservoir Rock and Fluid Transport Properties, second ed., Gulf Professional Publishing, 2004.

[25] A.P. Byrnes, E.K. Franseen, W.L. Watney, M.K. Dubois, The role of moldic porosity in Paleozoic Kansas reservoirs and the association of original depositional facies and early diagenesis with reservoir properties, Kansas Geological Survey, Open-file Report no. 2003-32, 2003.

[26] J.O. Amaefule, M. Altunbay, D. Tiab, D.G. Kersey, D.K. Keelan, Enhanced Reservoir Description: Using core and log data to identify Hydraulic (Flow) Units and predict permeability in uncored intervals/wells, in: SPE 26436 presented at 68th Ann. Tech. Conf. And Exhibit, Houston, TX, 1993. 